\title{
Atropello de vertebrados en una carretera secundaria en Costa Rica
}

\author{
Esteban Rojas Chacón \\ Centro de Rescate Tortufauna, La Garita de Alajuela, Costa Rica; esrocha88@yahoo.es
}

Recibido 23-VIII-2010 Corregido 6-IX-2010 Aceptado 17-IX-2010

\begin{abstract}
Vertebrate roadkills in a secondary road in Costa Rica. Roads have been one of the most significant anthropological changes in natural landscapes. Their effects are much more visible and strong in animal populations, which suffer from loss of connectivity, home range and habitat, and loss of individuals from roadkills, which is the most visible and easy to measure effect. From May 24 through July 28, 2010, I recorded all roadkills three times a week in a segment of 850 meters of a secondary road, in the Central Valley of Costa Rica. Nine animals were killed when the number of vehicles in the road was lower, compared with three when the number of vehicles increased. This negative correlation $(r$ $=-0,545)$ was unexpected. The most affected taxonomic group was the Bufonidae (Amphibia), with 5 roadkills.
\end{abstract}

\section{KEY WORDS}

Road ecology, roadkills, secondary road, flat fauna, Costa Rica.

\begin{abstract}
RESUMEN
Las carreteras han sido uno de los cambios antropológicos más notables dentro de los paisajes naturales. Sus efectos son mucho más visibles y fuertes en poblaciones animales, las cuales sufren pérdida de conectividad, disminución del ámbito de distribución y hábitat; y la disminución de individuos por muertes en carretera. De estos tres efectos, la muerte en carretera es el más visible y fácil de medir. En este estudio se tomó un registro tres veces por semana de todos los atropellos para un segmento de 800 metros de una carretera secundaria, durante un periodo de dos meses de 2010; en el Valle Central de Costa Rica. Nueve vertebrados fueron atropellados en un período donde la cantidad de vehículos fue menor, en comparación con tres atropellos cuando el tráfico fue mayor, generando una correlación negativa entre el número de muertes y el número de vehículos $(r=-0,545)$. El grupo taxonómico más afectado fue Bufonidae, con cinco atropellos.
\end{abstract}

\section{PALABRAS CLAVE}

Ecología de caminos, atropellos, caminos secundarios, "fauna aplastada", Costa Rica.
Las carreteras constituyen un factor antropológico que proveen servicios importantes pero que están teniendo una influencia en la hidrología, geomorfología y los procesos de los ecosistemas (Switalsky et al. 2004). Es por este último factor que se está desarrollando una nueva tendencia de la ecología del paisaje que trata del efecto ecológico de las carreteras sobre la zona donde se encuentran, llamada road ecology o ecología de caminos (Forman et al. 2003). El estudio de este tema ya ha sido tomado en cuenta anteriormente. En su clásica "guía de fauna aplastada" Knutson (1987) identificó las especies más afectadas en los EEUU y recapituló la información más importante en otras regiones del mundo.
Las consecuencias de las carreteras van desde la fragmentación de hábitats y pérdida de hábitat (Coffin 2007, Eigenbrod et al. 2008) hasta la posibilidad en plantas invasoras de colonizar las orillas de los caminos (Forman \& Alexander 1998). Además, las carreteras se pueden volver precursores de futuros impactos porque facilitan el desarrollo de la tierra, la expansión de la red vial (Riiters \& Wickhan 2003) y la proliferación de incendios forestales causados por el ser humano (Silva Matos et al. 2002).

Sus efectos son mucho más visibles y fuertes en poblaciones animales, las cuales sufren de: pérdida de conectividad pues las barreras que bloquean el paso de animales forman poblaciones más pequeñas, disminución del ámbito de distribución ya que se da una evitación por parte 
de la especie debido al efecto del ruido producido por la carretera y la disminución de individuos por las muertes en carretera (Forman \& Alexander 1998).

De estos tres efectos, la muerte en carretera (también llamados atropellos o atropellamientos) es el más visible (Bissonette \& Rosa 2009) y fácil de medir. Según Forman \& Alexander (1998) en las últimas tres décadas los atropellos han superado a la cacería como la causa directa de mortalidad de vertebrados en tierra por parte del ser humano, y en Costa Rica afecta a miles de especies por año (MongeNájera 1996).

Los estudios sobre este tema se han dado más que todo en zonas no tropicales, principalmente en Estados Unidos y Canadá donde se ha identificado que los impactos de las carreteras en los animales ameritan más investigación y que se tomen medidas a la hora de hacer las carreteras y en las ya existentes (Fahrig \& Rytwinski 2009). En Costa Rica se ha realizado investigación sobre el efecto de las carreteras (Monge-Nájera 1996), siendo pionera en los trópicos, sin embargo se necesita más información sobre este tema.

Este es el segundo estudio de campo publicado de Costa Rica, y el primero en el país en comparar un periodo de mucho tráfico con uno de poco tráfico.

\section{METODOLOGÍA}

Se analizó un trayecto de $850 \mathrm{~m}$ de carretera nacional (ruta 136) la cual comunica los distritos de Garita y Turrúcares de Alajuela y además es un punto de conexión de la carretera San José-Orotina (ruta 3) con la carretera San José- Caldera (ruta 27), inaugurada a inicios de 2010.

Se realizaron tres recorridos por semana desde el 24 de mayo hasta el 28 de julio de 2010, en los que se tomó registro de todos los vertebrados muertos en la carretera y sus orillas, que presentaran lesiones evidentes de un atropello(aplastadas, con órganos expuestos). En los casos posibles se identificó el individuo a nivel de especie ya que en ocasiones los animales quedan en un estado que dificulta la identificación de la especie (Aroyave \& Gómez 2006). Además se registró la cantidad de vehículos (automóviles, motocicletas, furgones) que pasaron por el lugar por un tiempo de diez minutos.

Se planteó la hipótesis de que se daría una correlación positiva entre la cantidad de vehículos y el número de muertes.

Este ejercicio se llevó a cabo analizando solamente la variable de muertes y de tráfico en carreteras debido a la poca disponibilidad de tiempo y a que estas variables son las más fáciles de medir, en comparación con otras variables que se toman en cuenta en estudios del efecto de carreteras en animales (densidad de población, tipo de camino, movilidad de especies, entre otros).

\section{RESULTADOS}

Trece vertebrados fueron registrados muertos: dos mamíferos, tres reptiles, tres aves y cinco anfibios. De los 16 recorridos, 11 resultaron en avistamientos $(68,75 \%)$; y solo se encontró en dos oportunidades más de un registro que nunca superó más de dos muertes (Cuadro 1).

El sapo común Chaunus marinus se encontró mayoritariamente en cinco oportunidades sin embargo solo en dos de las identificaciones se pudo comprobar que se trataba de esta especie y no de otra especie debido al mal estado del individuo. Después se identificaron dos palomas que por lo similar en su plumaje probablemente pertenecen a

CUADRO 1

Número de muertes y número de vehículos cada10 minutos en un trayecto de carretera de 850 metros

\begin{tabular}{|c|c|c|}
\hline $\begin{array}{l}\text { № de } \\
\text { vehículos } \\
\text { cada } 10 \\
\text { minutos }\end{array}$ & $\begin{array}{c}\text { Animales } \\
\text { muertos }\end{array}$ & $\begin{array}{c}\text { Grupo taxonómico } \\
\text { o especie }\end{array}$ \\
\hline 98 & 0 & - \\
\hline 93 & 1 & Chelydra serpentina \\
\hline 93 & 0 & - \\
\hline 82 & 1 & Columbidae \\
\hline 80 & 0 & - \\
\hline 76 & 1 & Columbidae \\
\hline 74 & 1 & Bufonidae \\
\hline 73 & 0 & - \\
\hline 72 & 1 & Serpentes \\
\hline 69 & 1 & Bufonidae \\
\hline 58 & 1 & Muridae \\
\hline 57 & 1 & Quiscalus mexicanus \\
\hline 56 & 0 & - \\
\hline 54 & 2 & Boidae; Didelphis \\
\hline 51 & 1 & Chaunus marinus \\
\hline 49 & 2 & $\begin{array}{l}\text { Chaunus marinus; } \\
\text { Bufonidae }\end{array}$ \\
\hline
\end{tabular}


la misma especie. Solo se encontró una tortuga muerta y un roedor, además de un marsupial.

El número de vehículos circulando en la carretera secundaria fue mayor durante el período de tiempo en que la carretera principal se encontró abierta $(\mathrm{t}=0,56 ; 14 \mathrm{gl}$; $p>0,05)$. Sin embargo la hipótesis planteada no fue correcta, ya que se dio una correlación negativa $(r=-0,545)$ entre el número de vehículos y el número de muertes.

\section{DISCUSIÓN}

El aumento de autos se debió a la reapertura de una carretera principal en la que fluyen muchos vehículos de los cuales algunos ingresan por la carretera analizada. Entre estos vehículos se da un aumento considerable en los furgones (no se trabajo esta variable) sin embargo, estos causan un ruido muy fuerte del cual hubo ausencia en el momento que la carretera estuvo cerrada.

Todos los animales que fueron encontrados atropellados ya se habían registrado en otros lugares como lo reporta Aroyave et al. (2006), en una revisión de diferentes taxones analizadas en diferentes estudios, solamente no se encontraron registros en Quiscalus mexicanus (zanate) o en ictéridos. Sin embargo, en Costa Rica Quiscalus mexicanus, Didelphis marsupialis (zorro pelón) y Chaunus marinus (sapo común) ya habían sido registrados atropellados (Monge-Nájera 1996).

Tanto tortugas como serpientes y anfibios (sapos) han sido registrados en muchas investigaciones como propensos a atropellos (Carr \& Fahrig 2001, Shine et al. 2004). Inclusive Steen et al. (2006) menciona que Chelydra serpentina es más propensa que otras tortugas a sufrir atropellos. Todos tienen en común que sus movimientos son lentos lo cual es una desventaja a la hora de cruzar una carretera (Carr \& Fahrig 2001, Coffin 2007).

Se cree que las muertes de Didelphis y $Q$. mexicanus se deben a que se mantuvieron en la carretera por motivos de alimentación. El primero se encontraba cerca de una boa grande muerta (también fue registrada) sobre la carretera, el aspecto de Didelphis era mucho menos golpeado por los autos que el de la boa por lo que se presume que al ser atropellada la boa, Didelphis estaba consumiéndola. Esta especie ha aumentado en áreas urbanas (Monge-Nájera 1996) lo que puede facilitar que sea atropellada y además esto confirma que los animales pueden ser atropellados cuando quieren consumir carroña (Harris \& Scheck 1991 citado por Coffin 2007). Q. mexicanus fue atropellado cerca de una bolsa de basura dejada por un camión recolector por lo que se supone que pudo estar buscando en la basura cuando fue impactado.
El caso del roedor es adverso a los estudios de roedores o mamíferos pequeños que tienden a no cruzar carreteras (Rytwinski \& Fahrig 2003, Mc Gregor et al. 2008).

No se registraron atropellos de Felis domesticus (gatos), ni de Canis familiaris (perros), siendo estas dos especies las más encontradas anteriormente (Monge-Nájera 1996).

La hipótesis no fue apoyada, pues la relación negativa de Pearson $(r=-0,5)$ indica que al aumentar los autos disminuyeron los atropellos. La situación en general se podría atribuir a que debido a la mayor cantidad de ruido provocado por los furgones, algunos animales presentaron el efecto de evitación de carretera (Forman \& Alexander 1998) que hace que estos se alejen de las carreteras y así se evitan los atropellos sin embargo, se requieren más datos en relación con esta posibilidad.

Las explicaciones para los atropellos varían dependiendo de la especie: si está dispuesta a cruzar el camino y si tiene una respuesta para lograr evitar los vehículos (Clevenger et al. 2002). Muchos animales cruzan las carreteras, pero aparentemente no hay estudios de comportamiento en animales silvestres que indiquen si estos pueden evitar efectivamente de alguna manera los vehículos.

La disminución de especies debido a los atropellos puede poner en riesgo a la población local. Varios estudios han registrado proporciones de sexos que tiende a ser sesgadas hacia machos o hacia hembras debido a que cierto género es más propenso a morir en carretera debido a su comportamiento. Por ejemplo, las tortugas acuáticas utilizan las orillas de los caminos para hacer sus nidos (Aresco 2005) muriendo en el intento o en el camino de regreso, lo que provoca que la proporción de hembras sea menor con respecto a los machos (Gibbs \& Steen 2005). Esto puede traer consecuencias a las generaciones futuras pues sin hembras las tasas de regeneración de la población podrían disminuir drásticamente.

También se deben tomar medidas en las que se pueda controlar la velocidad de los vehículos dentro de los parques nacionales para evitar atropellos. Esta medida se aplica en el Parque Nacional Tikal en Guatemala donde los visitantes deben reducir su velocidad dentro del parque y esto se regula con diferentes puestos de control (MongeNájera, com. pers. set. 2010)

\section{AGRADECIMIENTOS}

Se agradece la ayuda de Julián Monge-Nájera por los valiosos comentarios y comunicaciones personales para el enriquecimiento de este trabajo. 


\section{REFERENCIAS}

Aresco, M. 2005. The effect of sex-specific terrestrial movements and roads on the sex ratio of freshwater turtles. Biological Conservation 123: 37-44.

Aroyave, M. \& C. Gómez. 2006. Impactos de las carreteras sobre la fauna silvestre y sus principales medidas de manejo. Revista EIA 5: 45-57.

Carr, L. \& L. Fahrig. 2001. Effect of road traffic on two amphibians species of differing vagility. Conservation Biology 15: 1071-1078.

Bissonette, J. \& S. Rosa. 2009. Road Zone Effects in Small-Mammal Communities. Ecology and Society 14: 27.

Clevenger, A., B. Chruszczc \& K. Gunsoc. 2002. Spatial patterns and factors influencing small vertebrate fauna road-kill aggregations. Biological Conservation 109: 15-26.

Coffin, A. 2007. From roadkill to road ecology: A review of the ecological effects of roads. Journal of Transport Geography 15: 396-406.

Eigenbrod, F., S. Hecnar \& L. Fahrig. 2008. Accessible habitat: an improved measure of the effects of habitat loss and roads on wildlife populations. Landscape Ecology 23:159-168

Fahrig, L. \& T. Rytwinski. 2009. Effects of roads on animal abundance: an empirical review and synthesis. Ecology and Society $14: 21$.

Forman, R. \& L. Alexander. 1998. Roads and their major Ecological effects. The Annual Review of Ecology, Evolution, and Systematics 29:207-31.

Forman, R., D. Spearling, J. Bissonette, A. Clevenger, C. Cutshall, V: Dale, L. Fahrig, R. France, C. Goldman, K. Heanue, J. Jones, F. Swanson, T. Turrentine \& T. Winter. 2003. Road Ecology: Science and Solutions. Island ,Washington D.C., EEUU.
Gibbs, J. \& D. Steen. 2005. Trends in Sex Ratios of turtles in the United States: implications of road mortality. Conservation Biology 19: 552-556.

Knutson, R. M. 1987. Flattened Fauna: A field guide to common animals of roads, streets, and highways. Ten Speed, Berkeley, California, EEUU.

Mc Gregor, R., D. Bender \& L. Fahrig. 2008. Do small mammals avoid roads because of the traffic? Journal of Applied Ecology 45: 117-123.

Monge-Nájera, J. 1996. Vertebrate mortality in tropical highways: The Costa Rican case. Vida Silvestre Neotropica 5: 154-156.

Riiters, K. \& J. Wickhan. 2003. How far to the nearest road? Frontiers in Ecology and the Environment. 1:125-129.

Rytwinski, T. \& L. Fahrig. 2003. Effect of road density on abundance of white-footed mice. Landscape Ecology 22:1501-1512.

Shine, R., M. Lemaster, M. Wall, T. Langkilde \& R. Mason. 2004. Why Did the Snake Cross the Road? Effects of Roads on Movement and Location of Mates by Garter Snakes (Thamnophis sirtalis parietalis). Ecology and Society 9: 9.

Silva Matos. DM., CJF. Santos \& DR: Chevalier. 2002. Fire and restoration of the largest urban forest of the world in Rio de Janeiro City, Brazil. Urban Ecosystems 6: 151-161.

Steen, D., M. Aresco, S. Beilke, B. Compton, E. Condon, K. Dodd, H. Forrester, J. Gibbons, J. Greene, G. Johnson, T. Langen, M. Oldham, D. Oxier, R. Saumure, F. Schueler, J. Sleeman, L. Smith, J. Tucker \& J. Gibbs. 2006. Relative vulnerability of female turtles to road mortality. Animal Conservation 9: 269-273.

Switalsky, T., J. Bissonette, T. De Luca, C. Luce \& M. Madej. 2004. Benefits and impacts of road removal. Frontiers in Ecology and the Environment 2: 21-28. 